\title{
Teleradiology: results of a questionnaire of Cerman radiologists
}

\author{
R Bolte*, MWalz*, KJ Lehmann*, THothorn*, C Brill*, L Hothor" ${ }^{\dagger}$ and M Ceorgi* \\ *Department of Cinical Radiology, University of Heidelberg ${ }^{\dagger}$ LG Bioinformatics, University of Hannover, Cermany
}

\begin{abstract}
Summary
A questionnaire was sent by mail to 4400 radiologists (i.e. to at least 1000 radiological institutions). The response rate was about $5 \%$ The results showed that 47\% of respondents felt well informed about teleradiology, 49\% not enough and 3\%not at all. Image and report transfer as well as interfaces to reference databases, educational applications, technical quality surveillance and product support (maintenance) were considered to be increasingly important areas. Smaller institutions (13 doctors) judged expert consultation as more important than bigger institutions. Standardization, system stability and data security were demanded as well as guidelines, for example concerning the linking of report and image, correct documentation and required image quality. Technically most demands can be fulfilled today but this technology is not yet commonly induded in teleradiology systems.
\end{abstract}

The aims of the study were the analysis of the present and future requirements in teleradiology from the radiologists' point of view and the conception of a practicable and useful scenario for teleradiology applications in Germany and comparable countries.

\section{Methods}

A questionnaire was sent by mail to 4400 radiologists (i.e. to at least 1000 radiological institutions) at the beginning of February 1997. The addresses were taken from a database belonging to Schering, which gave German radiologist heads of department, senior radiologists and doctors in private consulting rooms. The contents of the questionnaire were worked out in cooperation with DRG (German Association of Radiologists, section on information techniques) and BVDRN (Occupational Association of German Radiologists and Nuclear Medicine, section on teleradiology).

\section{Results}

Over 200 radiologists sent the questionnaire back and 199 answers could be included in the statistical evaluation, representing nearly $5 \%$ of radiologists and $15-20 \%$ of the institutions. Several results such as the distribution of

Correspondence: Dr MWalz, Department of Cinical Radiology, Faculty of Cinical Medicine, University of Heidelberg, Kinikum Mannheim, Theodor-Kutzer-Ufer 1-3, D-68135 Mannheim, Cermany (Fax: +49621383 3817; Email:km22@umms.uni-mannheimde)

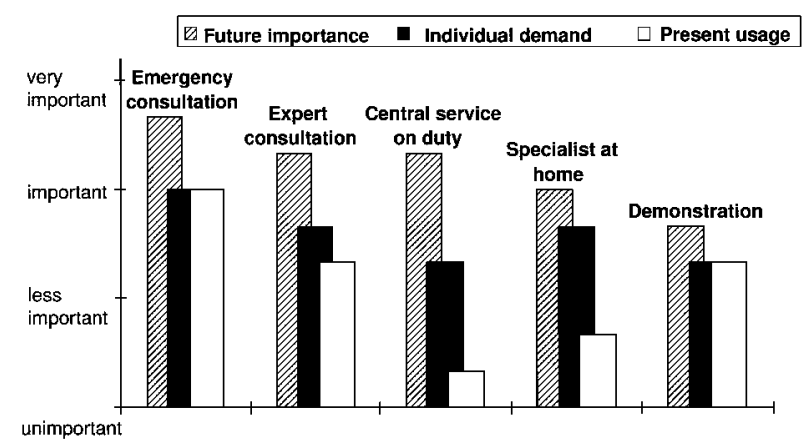

Fig 1 Importance of teleradiology application areas (part 1).

equipment (77\% had computerized tomography, 50\% magnetic resonance imaging, 87\% PCs, 75\% ISDN, 50\% Internet access, nearly 50\%workstations and networks, 30\%DICOM3) or size of the institutions were similar to known figures from other studies by industry or medical associations. On average each institution employed five doctors and 16.5 medical

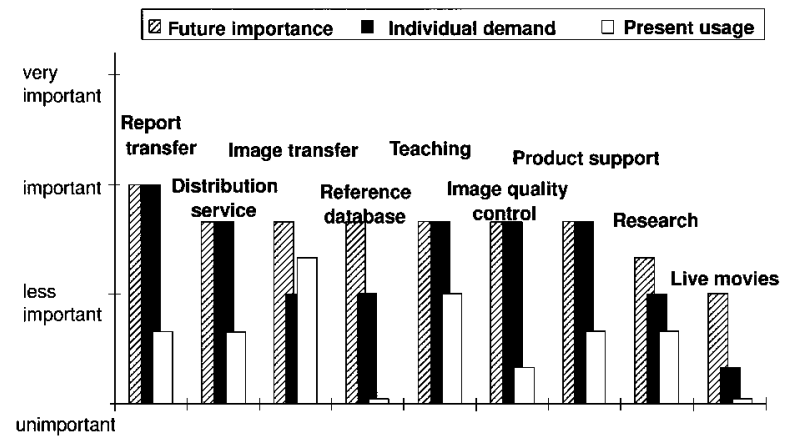

Fig 2 Importance of teleradiology application areas (part 2). 
R Bolte et al. Teleradiology

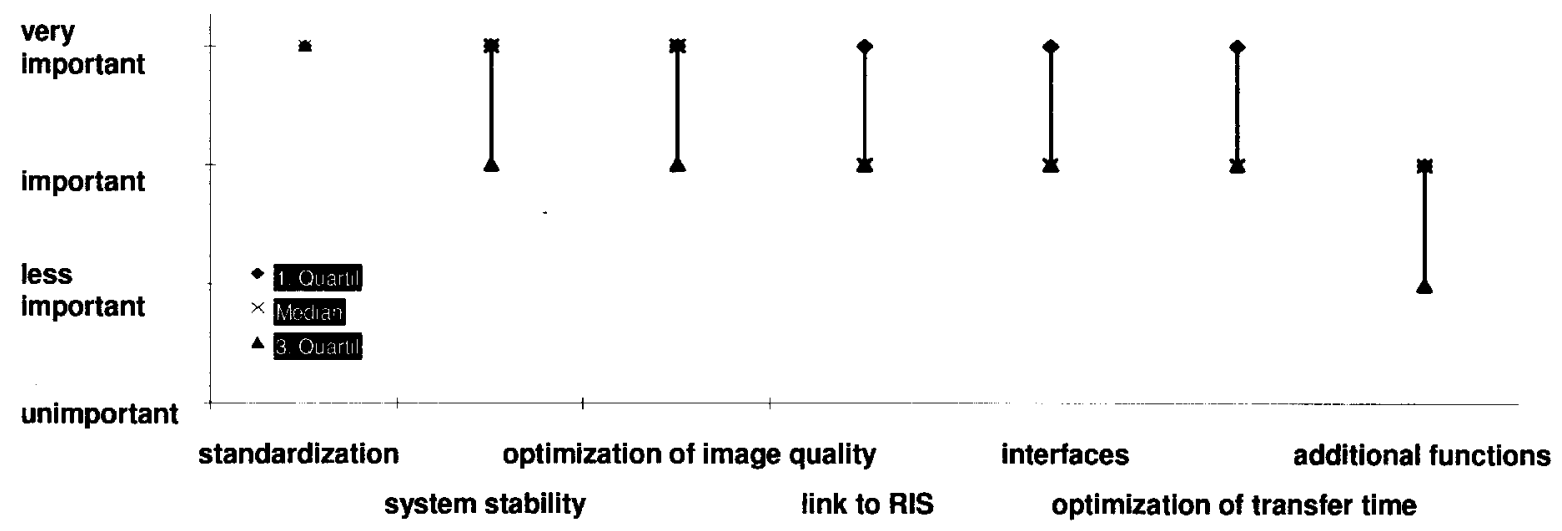

Fig 3 Importance of requirements for teleradiology concerning systems and equipment.

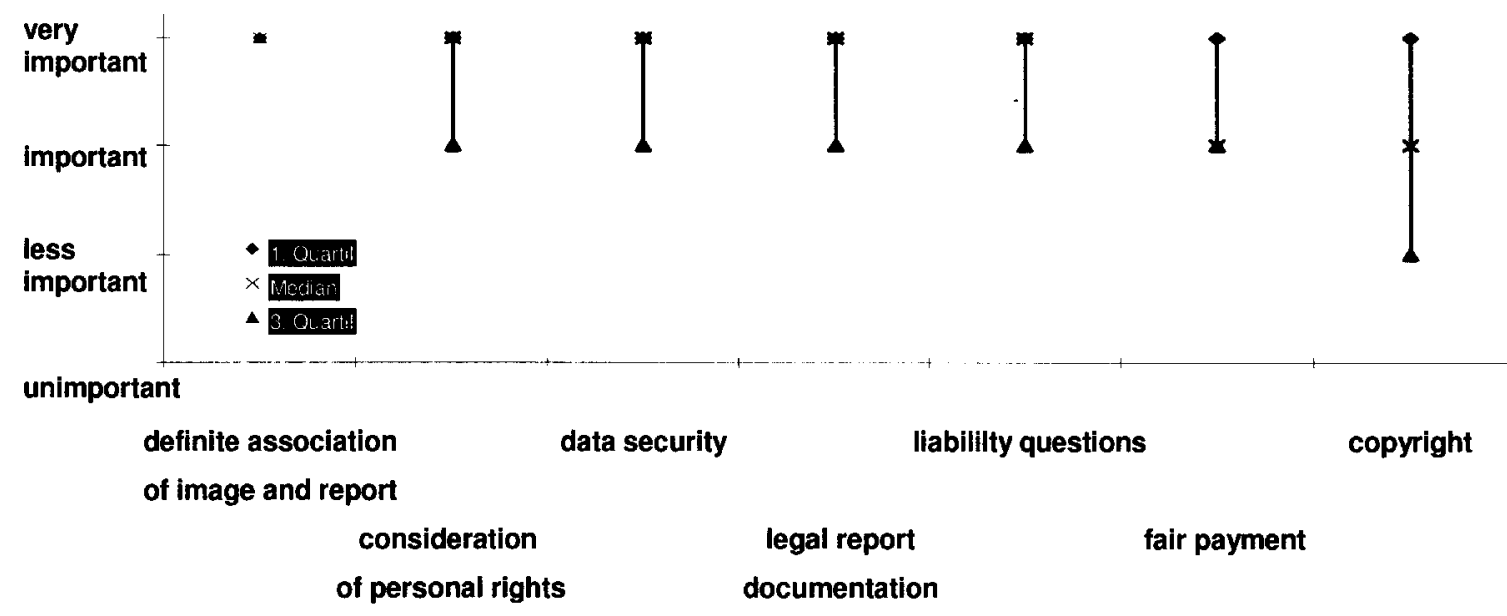

Fig 4 Importance of requirements for teleradiology concerning legal, organizational and vocational aspects.

technicians. Forty-eight per cent of answers originated from hospitals versus $52 \%$ from private consulting rooms. Fourteen per cent of answers represented the opinion of universities, while $32 \%$ were teleradiology users. These last figures probably overestimate the mean values. However, all these influences can be registered and separated by groupbased evaluation

Of the respondents, $47 \%$ felt well informed about teleradiology, 49\% not enough and 3\% not at all. Even among teleradiology users only $13 \%$ felt well informed. The main future application areas were emergency and expert consultation; more and more radiologist services were expected to be provided from home or central points (Fig 1).

Image and report transfer as well as interfaces to reference databases, educational applications, technical quality surveillance and product support (mintenance) were considered to be increasingly important areas (Fig 2). Smaller institutions (1-3 doctors) judged expert consultation as more important than bigger institutions. Image and report distribution services were of more interest to those in private consulting rooms.

\section{Discussion}

The main requirements for almost every radiologist are standardization of the systems, so that the systems can communicate with each other, system stability, which saves time, costs and sometimes even lives, and good image quality (Fig 3). Links to radiology information systems and picture archiving and communications systems (PACS) were considered especially important by those who work with these systems (60\% rated themvery important versus 33\% for those without PACS). From a medicolegal point of view there was a demand for the strongest links between report and image, appropriate data security, documentation and liability questions (Fig 4). The introduction of fair payments was mainly a matter of concern to radiologists in private consulting rooms. The respondents also had views on image quality (Fig 5): $68 \%$ demanded at least a comparable quality to the conventional hard copy, while $32 \%$ asked for the original quality, which nowadays means transfer of the digital data from the modality to the local teleradiology system and further to 


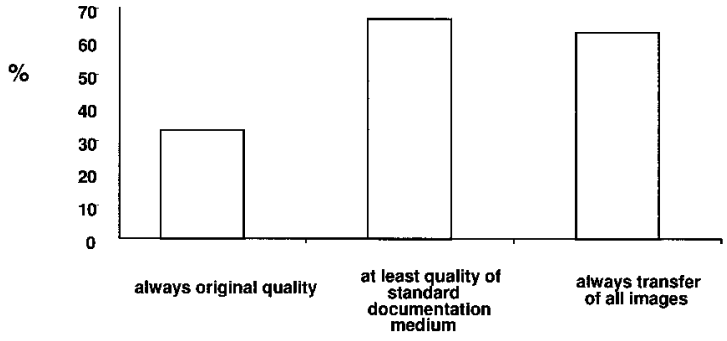

Fig 5 Requirements for image quality for primary reporting. discussed and solutions provided before the majority will use teleradiology. It must be recognized that there exist many different application areas with different requirements. Local conditions and interests of radiologists - and other medical or non-medical groups - are different too. However, standardization, systemstability and data security are being demanded, along with guidelines, for example for the linking of report and image, correct documentation and image quality, as well as political activities. These are presently the most urgent tasks in teleradiology from German radiologists' point of view.

Further information about this study is available on the Internet ${ }^{1}$.

Acknowledgements: The survey was funded by the German government represented by $\mathrm{BMBF}$ and $\mathrm{DFN}$.

\section{Reference}

1 http://www.rzuser.uni-heidelberg.de/ n17/index.html another teleradiology system with high visualization quality. Most radiologists thought that lossy compression should be allowed if no loss of relevant information occurs - although this is difficult to prove- and that all images should be transferred each time.

The present study shows the situation concerning teleradiology and the requirements of radiologists in Germany. Technically most demands can be fulfilled today but this technology is not yet commonly included in the systems. Other aspects such as legal or financial requirements must be

\title{
The experience of the Royal Children's Hospital mental health service videoconferenaing project
}

\author{
Harry Celber
}

Mental Health Senvice, Royal Children's Hospital, Melbourne, Australia

\begin{abstract}
Summary
In April 1995 the Royal Children's Hospital Mental Health Service in Malbourne piloted the use of videoconferencing in providing access for rural service providers and their dients to specialist child and adolescent psychiatric input. What began as a pilot project has in twoyears become integrated into the service-delivery systemfor rural Victoria. The experience of the service in piloting and integrating the use of videoconferencing to rural Victoria has been an important development for child and adolescent mental health senvices in Australia.
\end{abstract}

The emergence of telemedicine in Australia is an important development in the delivery of health care. The main

Correspondence: Mr H Celber, Travencore Center, 50 Flemington Street, Flemington, Melbourne, Victoria 3031, Australia (Fax: +61 39345 6010; Email: goldben@deakin.edu.au) applications have been in psychiatry, with the majority of the present 60-80 videoconferencing health sites nationwide being developed for the use of that cinical specialty ${ }^{1}$. In April 1995 the Royal Children's Hospital mental health service (ROHMHS) in Melbourne, Australia, piloted the use of videoconferencing to facilitate access for rural service providers and their clients to specialist child and adolescent 